\title{
Exercise rehabilitation new genre pioneering
}

\author{
Chang-Ju Kim (iD https://orcid.org/0000-0003-4749-5795
}

In the volume 14, issue 3 of the Journal of Exercise Rebabilitation (JER), various studies from many countries have been published, and a new genre of research has been included. It is very meaningful to pioneer new areas of exercise rehabilitation for the development of exercise rehabilitation.

The basic medical experiments of exercise rehabilitation on the diseases, such as "the effects of treadmill exercise on the CREP/ $\mathrm{BDNF} / \mathrm{TrkB}$ signaling pathway in traumatic brain injury," "the effects of treadmill exercise after marrow stromal cell transplantation in spinal cord injury," and "altitude exercise training damages small intestinal mucosa barrier injury," have been published. Researches on the mechanisms of exercise rehabilitation and diseases are essential for the scientific establishment of exercise rehabilitation.

The studies of "the effect of neurodevelopmental treatment on the rehabilitative medical examination in the urology area" and "the effects of neurodevelopmental treatment on the growth of premature infants in the neonatal intensive care unit" are a new research topic of exercise rehabilitation. The application of exercise rehabilitation in various clinical departments is a challenge for the development of medicine.

In particular, a study of "the effects of muscle relaxation techniques on anxiety and depression in leprosy patients" and a study of "the effects of combined and conventional exercise trainings in stroke patients" are considered of great clinical and social significance.

Many research results associated with rehabilitation, such as skin temperature, posture, estrogen, pain, are included in this is- sue. It is also interesting that the studies of baseball pitching were carried out simultaneously in Korea and Japan.

As articles of "psychological skills training for Paralympic table tennis athletes," "therapeutic recreation based on partner and group activities," and "environmental constraints within hospitals on physical activity level of cancer patients," have been published, the scope of JER has expanded to the "Humanities and Social Sciences" area.

In this issue, a wide range of studies of exercise rehabilitation have been published, and these studies are in line with the aims and scope of the JER that states "from basic medicine to clinical applications." This situation is considered to be very positive for pioneering new areas of exercise rehabilitation. I hope that JER will continue to develop new researches on exercise rehabilitation beyond existing prevention and treatment.

\section{CONFLICT OF INTEREST}

No potential conflict of interest relevant to this article was reported.

President of the Korean Society of Exercise Rehabilitation Department of Physiology, College of Medicine, Kyung Hee University, 26 Kyungheedae-ro, Dongdaemun-gu, Seoul 02447, Korea E-mail: changju@khu.ac.kr 